\title{
Nonabsorbable polymer clip as a cause of chronic perineal pain after radical prostatectomy: Description of a case treated by transperineal approach
}

\author{
Massimiliano Creta $^{1}$, Sergio Di Meo ${ }^{1}$, Roberto Buonopane ${ }^{1}$, Bernardo Rocco ${ }^{2}$, Vittorio Imperatore ${ }^{1}$ \\ ${ }^{1}$ Unità Operativa di Urologia, Buon Consiglio Fatebenefratelli Hospital, Napoli, Italy; \\ ${ }^{2}$ UOC di Urologia, Fondazione IRCCS Ca' Granda Ospedale Maggiore Policlinico, Milano, Italy.
}

\begin{abstract}
Summary The occurrence of persistent perineal pain caused by surgical clips has rarely been described after radical prostatectomy (RP). We describe the case of a patient complaining of chronic perineal pain occurred soon after robotic RP, refractory to conventional medical therapy and exacerbated by the sitting position. Pain was related to a nonabsorbable polymer clip used to secure lateral pedicles. A transpeerineal approach was used to perform an hydrodissection of the rectovesical space at the level of the surgical clip combined with local injection of mepivacaine and betametasone. The patient experienced a clinically significant reduction of pain that remained stable at three months' follow-up.
\end{abstract}

KEY WORDS: Pain; Prostatic neoplasms; Prostatectomy.

Submitted 3 September 2016; Accepted 26 November 2016

\begin{abstract}
INTRODUCTION
Postoperative pelvic pain is expected after radical prostatectomy (RP) (1). However, pain lasting more than 6 months is uncommon (1). Differential diagnosis is vast and includes chronic pelvic pain syndrome, urolithiasis, retained foreign body, pelvic joint dysfunction, surgical clip migration and erosion at the level of the urethrovesical anastomosis $(1,2)$. There is no previous report of perineal pain caused by normally anchored surgical clips. Herein, we present a case of persistent intense post-RP perineal pain caused by a nonabsorbable polymer clips normally anchored along a neurovascular bundle far from the urethrovesical anastomosis and successfully treated with transperineal local hydrodissection and injection of anesthetic and corticosteroids.
\end{abstract}

\section{Case report}

A 66-years old man was referred to our Urology Unit complaining of chronic perineal pain unresponsive to conventional medical therapy with nonsteroidal antiinflammatory drugs and gabapentin. His past surgical history was relevant for robotic RP performed 10 months before for a Gleason 3+4 prostate cancer while his past medical history was unremarkable. Onset of pain was in the early post-operative course of RP and it worsened in the sitting position. Maximum pain intensity was 8 on a Visual Analogue Scale (VAS) 0 to 10. The patient denied any Lower Urinary Tract Symptom, macroscopic hematuria and fewer. Physical examination of the abdomen and the perineum was unremarkable. Digital rectal examination excluded local prostate cancer recurrence. However, a nonabsorbable polymer clip could be appreciated along the left neurovascular bundle, far from the urethrovesical anastomosis and finger pressure applied on it was able to elicit and intensify the pain described by the patient.

Patient post-operative Prostate Specific Antigen was undetectable. Findings from laboratory assessments and abdominal ultrasound were unremarkable. We hypothesized that pain could be secondary to a nerve entrapment syndrome caused by the surgical clip at the level of the left pelvic neurovascular bundle.

The patient was hospitalized and we decided to manage it based on evidences from scientific literature in the orthopedic field (3). Accordingly, we used a transperineal approach to perform an hydrodissection of the rectovesical space at the level of the surgical clip combined with local injection of mepivacaine and betametasone. In detail, the procedure was performed under spinal anesthesia with the patient in the lithotomy position. A18 Gauge biopsy needle was used for saline $(10 \mathrm{~mL})$ injection in order to obtain hydrodissection of the entrapped neurovascular bundle followed by local injection of 10 $\mathrm{mL}$ of mepivacaine $20 \mathrm{mg} / \mathrm{mL}$ combined with $2 \mathrm{~mL}$ of betametasone $4 \mathrm{mg} / 2 \mathrm{~mL}$.

The procedure was performed under digital and transrectal ultrasound guidance in order to localize the site of injection (Figure 1).

The procedure was uneventful and the patient was discharged home on post-operative day 1 . On post-operative day 1 pain intensity reported by the patient was 2 on a VAS 0-10 and on post operative day 5 pain intensity was 4. At 3 months follow up pain intensity remained stable (VAS score:4) and the patient declared to be satisfied. Finger pressure on the site of the clip during digital rectal examination was unable to intensify pain intensity. 
Figure 1.

Sonographic anatomy for the injection technique described. B: Bladder, N: Bladder neck, C: Surgical clip, $\mathrm{H}$ : Hydrodissection space.

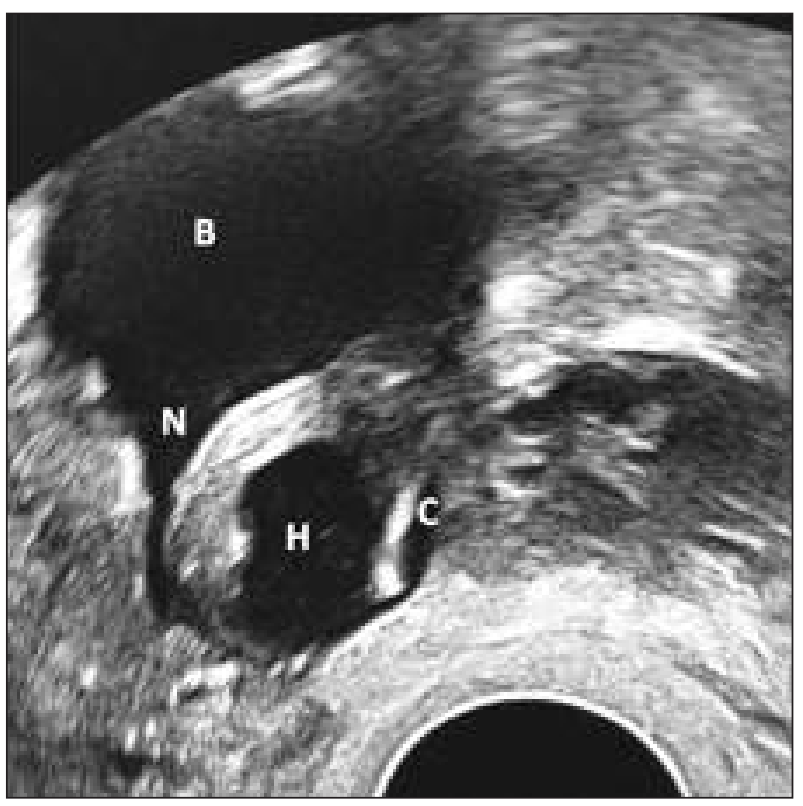

\section{Discussion}

Surgical clips are commonly used during laparoscopic and robotic RP in order to secure lateral pedicles by avoiding thermal injury to the neurovascular bundle (1-2). Complications related to the use of these clips have been reported and are mainly related to their migration and erosion into the urinary tract (1-2). Moreover, biologically inert foreign bodies are known to represent a substrate that can be colonized by biofilms (3). As consequence, Authors suggest to minimize the use of clips on tissue immediately adjacent to the anastomosis, and to make every effort to retrieve loose clips after the procedure (1-2). Patients most commonly present with urinary retention, obstructive lower urinary tract symptoms, hematuria, pyuria, dysuria, urgency, painful micturition (1). Surgical clips may be a source of pain. However, the occurrence of perineal pain related to surgical clips has rarely been described after RP (1).
Palou J et al. reported the case of a patient with severe perineal pain occurred soon after RP that prevented him from sitting normally (2). Pain was secondary to a metal clip protruding into the urethra through the urethrovesical anastomosis (2). To our knowledge, we described for the first time the occurrence of severe and persistent post-RP perineal pain caused by a nonabsorbable polymer clip used to secure lateral pedicles and the technique of transperineal management of this complication. Hydrodissection and corticosteroid injection are described in the orthopedic field as a valid option in case of pain related to nerve entrapment (4). Hydrodissection dilate the injection space and can free the entrapped structures such as nerves. Given the rising number laparoscopic and robotic RP together with the growing use of nonabsorbable polymer clip in this field, the number of clip related complications, including that described in the present case report, is expected to increase. In our experience, this procedure is simple to perform, safe, and can be repeated in necessary.

\section{Conclusions}

The present case demonstrates that severe perineal pain may occur as a complication of nonabsorbable polymer clips at the level of neurovascular bundles after RP. The combination of local hydrodissection and injection of anesthetics and steroids through a transperineal approach may represent a valid strategy for patients unresponsive to medical therapy.

\section{References}

1. Yi JS, Kwak C, Kim HH, Ku JH. Surgical clip-related complications after radical prostatectomy. Korean J Urol. 2010; 51:683-7.

2. Palou J, Alberola JM, Villavicencio H, Vicente J. It's like a pain in th... perineum: a surgical clip protruding into the urethra through the urethrovesical anastomosis after radical prostatectomy. Scand J Urol Nephrol. 1997; 31:493-5.

3. Cennamo P, Montuori N, Trojsi G, et al. Biofilms in churches built in grottoes. Sci Total Environ. 2016; 543(Pt A):727-38.

4. Soneji N, Peng PW. Ultrasound-guided pain interventions - a review of techniques for peripheral nerves. Korean J Pain. 2013; 26:111-24.

\section{Correspondence}

Massimiliano Creta, MD

max.creta@gmail.com

Sergio Di Meo, MD

s.dimeo72@gmail.com

Roberto Buonopane, MD

robertobuonopane@libero.it

Vittorio Imperatore, MD (Corresponding Author)

v.imperatore@alice.it

Unità Operativa di Urologia, Buon Consiglio Fatebenefratelli Hospital

Via A. Manzoni, 220, 80123, Napoli, Italy

Bernardo Rocco, MD

bernardo.rocco@gmail.com

U.O.C. di Urologia, Fondazione IRCCS Ca' Granda

Ospedale Maggiore Policlinico

Via Commenda, 15, 20122 Milano (Italy 